\title{
Anesthetic Management of Abdominal Aortic Aneurysm Repair in a Patient With Tuberous Sclerosis Complex
}

\author{
Mohammed Hakim a, d, e, Shabana Z. Shafy ${ }^{\text {a, d }}$, Renee Henga, d, \\ Joseph D. Tobias ${ }^{\mathrm{a}, \mathrm{b}, \mathrm{c}, \mathrm{d}}$
}

\begin{abstract}
Tuberous sclerosis complex, also known as Bourneville disease, is an autosomal dominant, progressive neurocutaneous disorder with variable presentation and multisystem involvement. It is characterized by the development of benign tumors in multiple organs and tissues in the body, with the majority of patients having neurological involvement. We report a 19-year-old adolescent, with tuberous sclerosis and advanced systemic involvement of the disease, presenting for surgical repair of an abdominal aortic aneurysm. The etiology and presentation of TS are discussed and its multisystem organ involvement is reviewed with emphasis on the anesthetic implications of the disease.
\end{abstract}

Keywords: Tuberous sclerosis; Aortic aneurysm; Anesthesia

\section{Introduction}

Tuberous sclerosis (TS) is an autosomal dominant disorder with an incidence of approximately 1 in 5,000 - 10,000 live births. Along with neurofibromatosis and Sturge-Weber disease, it represents one of the three most common of the neurocutaneous disorders. Presentation varies due to incomplete penetrance and variable expressivity. It is linked to a mutation of the tuberous sclerosis complex 1 (TSC1) gene on chromosome 9 or the TSC2 gene on chromosome 16, both of these being tumor suppressor genes, which code for the proteins, hamartin and tuberin, respectively. Genetic testing for the pathogenic TSC1 or

Manuscript submitted June 10, 2019, accepted June 24, 2019

aDepartment of Anesthesiology \& Pain Medicine, Nationwide Children's Hospital, Columbus, OH, USA

bepartment of Pediatrics, The Ohio State University College of Medicine, Columbus, OH, USA

'Department of Anesthesiology \& Pain Medicine, The Ohio State University College of Medicine, Columbus, OH, USA

${ }^{\mathrm{d}}$ These authors contributed equally to this manuscript.

eCorresponding Author: Mohammed Hakim, Department of Anesthesiology \& Pain Medicine, Nationwide Children's Hospital, 700 Children's Drive, Columbus, OH 43205, USA.

Email: mohammed.hakim@nationwidechildrens.org

doi: https://doi.org/10.14740/jmc3326
TSC2 can be used to confirm the diagnosis. Recent studies have also demonstrated the significant role of the hamartin-tuberin complex in the pathogenesis of the disease through the inhibition of cellular signaling mediated by the mechanistic target of rapamycin (mTOR), which is an important regulator of cell cycle progression and protein synthesis $[1,2]$.

TSC is characterized by the development of benign tumors in multiple organs and tissues in the body with the potential for malignant transformation. The majority of patients present with signs and symptoms related to the central nervous system (CNS) including seizures, cognitive deficits, learning disabilities, behavioral problems and psychosocial difficulties. Collectively, these have been termed tuberous sclerosis-associated neuropsychiatric disorders (TANDs) [3, 4]. Dermatological features include hypopigmented macules also known as ash-leaf spots; shagreen patches; angiofibromas involving malar regions of the face; "confetti-like" macules distributed over the extremities; distinctive brown fibrous plaque on the forehead; and Ungal fibromas. Characteristic CNS lesions include subependymal giant cell astrocytomas, glioneuronal hamartomas (also called cortical tubers) and subependymal nodules [5, 6]. Renal angiomyolipomas are frequently seen. TSC can also present with pulmonary involvement known as lymphangioleiomyomatosis (LAM) that is indistinguishable from diffuse interstitial fibrosis [7]. Classical cardiac involvement is the rhabdomyoma, while mitral regurgitation and aortic aneurysms have also been reported [8-10]. Recent studies and reports have demonstrated an increased association of aortic aneurysm in patients with TSC $[9,10]$. We report a 19-year-old patient with TSC and advanced systemic involvement of the disease, presenting for surgical repair of an abdominal aortic aneurysm. The etiology and presentation of TS are discussed and its multisystem organ involvement is reviewed with emphasis on the anesthetic implications of the disease.

\section{Case Report}

The patient was a 19-year-old, 44.8-kg adolescent, who presented for surgical repair of an abdominal aortic aneurysm (AAA). The AAA was initially diagnosed at 13 years of age on a computed tomography (CT) imaging of the abdomen and pelvis, and had progressively increased in diameter. Her active medical problems included TS with advanced multisystem involvement including subglottic stenosis; scoliosis; lymphan- 
giectasia causing protein losing enteropathy; urinary incontinence; acquired hypothyroidism and hypoparathyroidism post thyroidectomy; hypoalbuminemia; retroperitoneal lipoma; possible residual intracardiac rhabdomyoma on the mitral valve; deep vein thrombosis (DVT) of the superior venous system (on chronic prophylactic anticoagulant therapy); anemia; vitamin D deficiency; and seizures with complex partial epilepsy and developmental delay. Her past medical history was significant for a cardiac rhabdomyoma; malignant chondroma; lymphedema of the left arm; Hurthle cell carcinoma of the thyroid; lymphangioma of small intestine; tethered cord malformation sequence; renal cysts; ureteral reflux and reactive airway disease. Past surgical history included craniotomy for resection of a subependymal giant cell astrocytoma; resection of a chondroma of the coccyx; incision and drainage of a perineal abscess; total thyroidectomy; insertion of a single lumen central venous port; and thoracoscopy with partial pleurectomy for recurrent accumulation of chylous fluid. Preoperative laboratory investigations showed an international normalized ratio (INR) of 1.2, prothrombin time of $14.7 \mathrm{~s}$ and activated partial thromboplastin time (aPTT) of 29 s. Renal function tests and electrolytes were within normal limits with a blood urea nitrogen (BUN) of $14 \mathrm{mg} / \mathrm{dL}$ and creatinine of 0.48 $\mathrm{mg} / \mathrm{dL}$. The hematocrit was $33 \%$ and the hemoglobin was 11.2 $\mathrm{g} / \mathrm{dL}$. Echocardiography demonstrated a small echogenic area in the anterolateral mitral valve papillary muscle suggestive of a residual rhabdomyoma, with normal cardiac anatomy and ventricular function. The most recent $\mathrm{CT}$ angiography of the abdomen and pelvis demonstrated the AAA with associated stenosis of the infrarenal abdominal aorta above the aneurysm. The aneurysm had increased in size compared to previous imaging, performed a year ago. There was high-grade stenosis or complete occlusion of the abdominal aorta at the inferior margin of aneurysm with no apparent connection between the aneurysm and the common iliac arteries. The iliac arteries were very small but patent, with normal branching. The imaging also demonstrated a stable retroperitoneal lipoma with mass effect on the inferior vena cava, which might have also been contributing to the severe stenosis of the aorta below the aneurysm. Allergies included loratidine, diphenhydramine and iron dextran complex. Current medications included warfarin ( $2 \mathrm{mg}$, once daily), albumin $25 \%$ intravenous injection (once a week), levothyroxine (300 $\mu \mathrm{g}$, once daily), calcium supplements $(1,000 \mathrm{mg}$, three times a day), calcitriol $(0.75 \mu \mathrm{g}$, once daily), ergocalciferol oral drops (40,000 units daily), prednisone $(2.5 \mathrm{mg}$, prior to intravenous immunoglobulin or sodium ferric gluconate), lamotrigine (150 mg, twice daily), norethindrone (5 mg, once daily), enalapril ( $2.5 \mathrm{mg}$, once daily), albuterol inhalational solution $(2.5 \mathrm{mg}$, inhaled by nebulizer as needed), diazepam ( $5 \mathrm{mg}$, once daily), ferrous fumarate (325 $\mathrm{mg}$, once daily), trimethoprim-sulfamethoxazole (twice a day on Saturday and Sunday), loperamide (2 mg, up to 4 doses/ day), ascorbic acid (500 mg, once daily), desloratidine (5 mg, once daily), ranitidine (300 mg, twice daily), lactobacillus probiotics and multivitamin supplements.

Physical examination demonstrated a Mallampati class I airway, history of subglottic stenosis with no symptoms at rest and right upper extremity restriction due to chronic lymphedema with use of a compression sleeve. The remainder of the physi- cal examination and preoperative vital signs were unremarkable. The anesthetic assessment and plan included an American Society of Anesthesiologists (ASA) physical classification III with a plan for a general anesthesia with invasive hemodynamic monitoring. The anesthetic plan, risks and benefits of general and regional anesthesia were discussed with the parent, and informed consent was obtained. Warfarin and enalapril were held 7 days and 1 day prior to the surgery, respectively. The patient was held nil per os for $8 \mathrm{~h}$ and transported to the operating room. After arrival in the operating room, the patient was placed in the supine position and routine ASA monitors were placed. The patient was allowed to breathe $70 \%$ nitrous oxide in oxygen for 3 min followed by placement of two peripheral intravenous catheters (14- and 20-gauge) using ultrasound guidance. Propofol $(180 \mathrm{mg})$, lidocaine $(40 \mathrm{mg})$, fentanyl $(100 \mu \mathrm{g})$ and rocuronium (50 mg) were administered intravenously followed by direct laryngoscopy. The patient's trachea was intubated with a $4.0 \mathrm{~mm}$ cuffed endotracheal tube (ETT) as a $4.5 \mathrm{~mm}$ ETT could not be placed to the pre-existing subglottic stenosis. A 22-gauge arterial line was placed under aseptic conditions in the right radial artery. Maintenance anesthesia included sevoflurane $(2-3 \%)$ in air/oxygen and intermittent doses of ketamine $(0.2 \mathrm{mg} / \mathrm{kg}$ every 1 - 2 h). Neuromuscular blockade was provided by intermittent doses of rocuronium. Intraoperative electrolyte and acid-base status were intermittently monitored and sodium bicarbonate and calcium chloride were administered as needed to maintain homeostasis. Intraoperative antibiotic prophylaxis included cefazolin. Additional intraoperative medications included dexamethasone $(12 \mathrm{mg})$ and heparin $(6,000$ units) prior to aortic cross-clamping.

The procedure involved open repair of the AAA with placement of an 8-mm polytetrafluoroethylene graft, sewn from the infrarenal aorta to its bifurcation. Following placement, there was Doppler confirmation of bilateral signals in the posterior tibial and the common femoral arteries. The procedure lasted approximately $7 \mathrm{~h}$ and progressed without incident. Total fluids included $5 \%$ albumin $(875 \mathrm{~mL}), 900 \mathrm{~mL}$ of normal saline and one unit of packed red blood cells. Intraoperative urine output was $155 \mathrm{~mL}$ and blood loss was $700 \mathrm{~mL}$. Prior to completion of the surgical procedure, hydromorphone (total dose of $0.7 \mathrm{mg}$ ) and acetaminophen $(600 \mathrm{mg})$ were administered followed by nurse-controlled analgesia with hydromorphone. Residual neuromuscular blockade was reversed with sugammadex $(200 \mathrm{mg})$ and the patient's trachea was extubated after the return of normal spontaneous ventilation with an adequate tidal volume. The patient was transported to the cardiothoracic intensive care unit (CTICU) in stable condition, where she remained for 2 days for postoperative hemodynamic monitoring. Enalapril was re-started on the second postoperative day for control of hypertension. She was discharged home a week later, with follow-up appointments by hematology and vascular surgery after 1 and 3 weeks, respectively. Her post-discharge course was unremarkable.

\section{Discussion}

Aneurysmal diseases in children can be characterized as true 
aneurysms associated with arterial infections, giant cell aortoarteritis, autoimmune connective tissue disease, Kawasaki's disease, Ehlers-Danlos syndrome or Marfan's syndrome, other forms of non-inflammatory medial degeneration, arterial dysplasias, congenital-idiopathic factors, as well as false aneurysms associated with extravascular events causing vessel wall injury or disruption. Although not often considered as a distinctive feature of TS, there have been reports of AAAs being an associated disorder, and are seemingly more common than stenotic diseases $[9,10]$. The diameter of a normal aorta varies with age, gender and body habitus, but for the majority of the patients an infrarenal aorta with diameter $\geq 3 \mathrm{~cm}$ is considered as aneurysmal and may require surgical intervention [11]. In TS, the pathogenesis of aortic aneurysm formation has been proposed to be the result of a defect in the connective tissue, similar to the loss of elastin fibers as seen in Marfan's syndrome [12]. Rolfes et al noted an association of TSC with fibromuscular disease and suggested that vascular dysplasia/ hyperplasia may be a manifestation of underlying hamartomatous formation of the vasculature [13].

Aortic aneurysms are often classified relative to their relationship with the renal vessels, with infrarenal being the most common point of origin. Presentation may vary from asymptomatic to primary clinical features such as abdominal/ back pain, palpable pulsatile abdominal masses or secondary symptoms arising from limb ischemia or compression leading to renovascular hypertension and bowel obstruction. Rupture is the most fatal complication, presenting with severe pain, hypotension and cardiovascular collapse [13]. Detailed physical examination with complete peripheral arterial vasculature assessment, along with diagnostic tests such as ultrasonography or CT imaging, can provide a diagnosis prior to clinical presentation. Management varies depending on the presentation and size of the aneurysm as well as the progress of the aneurysmal dilatation. Options for intervention range from open surgical or endovascular repair to conservative management with regular surveillance and assessment of an asymptomatic non-ruptured aneurysm. Joswiak et al proposed the possible application of non-surgical treatment of TS with rapamycin [14]. Rapamycin, also known as sirolimus, is an antibiotic derived from a bacterium (Streptomyces hygroscopicus). It is used as an immunosuppressive agent in organ transplantation. It normalizes the dysregulated mTOR pathway in cells deficient in either wild-type TSC1 or TSC2, and has resulted in beneficial therapeutic effects in patients with astrocytomas, lymphangioleiomyomatosis and renal angiomyolipomas. Further studies are needed to assess the effectiveness of this drug in preventing the development of other manifestations of TSC [15-17].

Patients with TSC may present for surgical treatment of the various associated disorders associated with the primary disease process. As with any surgical intervention, care begins with a thorough pre-anesthetic evaluation to identify the end-organ impact of the primary disease. Since behavioral problems and cognitive deficits are often concomitantly seen in these patients, communication and evaluation can be challenging during the perioperative course. Preoperative investigation should be guided by the past medical history and include a chest radiograph, 12-lead electrocardiogram and echocardiography to rule out anatomical cardiac lesions (cardiac rhabdomyoma) and conduction defects. Preoperative laboratory investigation should include a complete blood count, coagulation function with platelet count, renal and hepatic function tests, serum electrolytes and anticonvulsant medication levels. Routine anticonvulsant medications should be administered throughout the perioperative period, on the morning of the procedure, with subsequent intraoperative and postoperative dosing as needed [18]. Pre-medication with a benzodiazepine (oral midazolam) or intranasal dexmedetomidine may help reduce anxiety and facilitate inhalational induction as needed. Parental presence in the operating room may assist with the care of the child during anesthetic induction and may further reduce anxiety. Based on the patient's associated co-morbid conditions, placement of a peripheral intravenous cannula with intravenous induction may be chosen as was done for our patient. This was easily accomplished with nitrous oxide sedation and topical analgesia. Intravenous induction agents such as propofol can be used in the absence of significant cardiac involvement, with consideration of the use of etomidate if myocardial contractility is affected. Sevoflurane and nitrous oxide may be used for inhalational induction with various options available for maintenance anesthesia (volatile-anesthetic based or total-intravenous anesthesia with propofol-remifentanil or sufentanil) depending on the patient's hemodynamic status and the duration of the case. In the adult population, anecdotal reports have demonstrated the safe and effective implementation of regional or neuraxial anesthetic techniques with a shorter length of stay and lesser rates of cardiopulmonary morbidities [19-21].

Oral manifestations of TSC include dental enamel pitting, gingival fibromas, gingival hyperplasia, hemangiomas, nodular tumors, papillomas, pseudocystic lesions of the mandible and thickening of the alveolar bone. Airway management may be challenging due to the presence of oropharyngeal or laryngeal tumors obscuring the larynx and interfering with endotracheal intubation for anesthetic management of airway. Tongue base hamartomas necessitating fiber-optic intubations have also been reported $[22,23]$. In our patient, history and physical examination did not reveal any concerns for airway management.

Various CNS lesions have been described in TSC including giant cell astrocytomas, cortical tubers and subependymal nodules. At times, these may be asymptomatic. Preoperative review of previous CNS imaging is indicated. These CNS lesions may enlarge and present with features suggestive of hydrocephalus with focal neurological deficits, increased intracranial pressure, seizures and behavioral disorders [6].

Cardiac signs and symptoms may be due to anatomical lesions (cardiac rhabdomyoma) or associated conduction and rhythm disturbances (atrial and ventricular tachycardia, supra-ventricular fibrillation and complete heart block). Recent studies have demonstrated the association of TSC with WolfParkinson-White syndrome. Routine preoperative 12-lead electrocardiography and echocardiography is suggested along with continuous intraoperative electrocardiography [24]. Depending on the patient's clinical history, monitoring of lead II and $\mathrm{V}_{5}$ may be indicated to increase arrhythmia identification. The association of mitral valve regurgitation and thoracic/ab- 
Table 1. Previous Reports of Abdominal Aortic Aneurysm in Tuberous Sclerosis

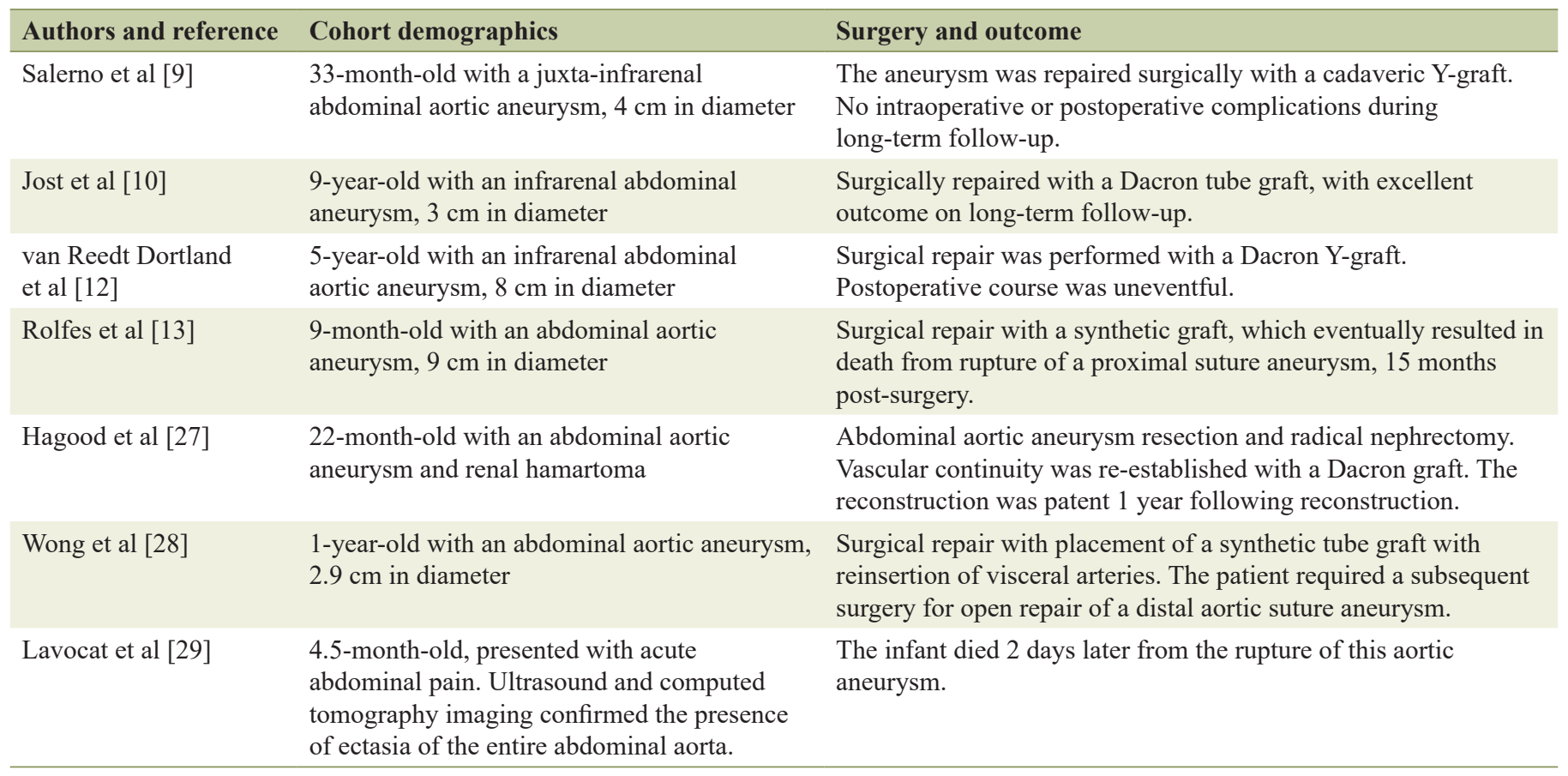

dominal aneurysms has been in TSC. Echocardiography and magnetic resonance imaging (MRI) are important non-invasive investigations for identification of these anatomical abnormalities especially when patients are asymptomatic [8-10].

Pulmonary involvement is rare in TSC, but can present with LAM and multifocal micronodular pneumocyte hyperplasia (MMPH), with symptoms including dyspnea, hemoptysis, recurrent pneumothorax and pulmonary hypertension. LAM is characterized by abnormal proliferation of smooth muscle cells, leading to cystic lung lesions and spontaneous pneumothorax $[7,25]$. As indicated by the history and physical examination, chest radiography and pulmonary function testing may be indicated. Intraoperatively implementation of appropriate lung protective measures may be indicated in patients with pulmonary parenchymal involvement [26].

In summary, various anecdotal reports have noted the association of aortic aneurysms in patients with TSC (Table 1) [9, 10, 12, 13, 27-29]. Early and periodic screening for aortic aneurysms in patients with TSC can help establish an early diagnosis, identify progression and offer timely surgical intervention to prevent complications. As noted in the anecdotal reports, surgical intervention is generally effective in resection of the aneurysm and prevention of late complications including rupture. In addition to the usual anesthetic concerns involved with AAA resection, preoperative identification and optimization of end-organ involvement of TSC may facilitate the perioperative care of these patients [30-32].

\section{Acknowledgments}

None.

\section{Financial Disclosure}

None.

\section{Conflict of Interest}

None.

\section{Informed Consent}

We have obtained informed consent from our patient.

\section{Author Contributions}

Mohammed Hakim wrote the paper. Shabana Zainab Shafy wrote the paper and edited it. Renee M. Heng took part in the clinical care of the patient. Joseph D. Tobias did the final approval of the paper and reviewed it.

\section{References}

1. Curatolo P, Bombardieri R, Jozwiak S. Tuberous sclerosis. Lancet. 2008;372(9639):657-668.

2. Tee AR, Fingar DC, Manning BD, Kwiatkowski DJ, Cantley LC, Blenis J. Tuberous sclerosis complex-1 and -2 gene products function together to inhibit mammalian target of rapamycin (mTOR)-mediated downstream 
signaling. Proc Natl Acad Sci U S A. 2002;99(21):1357113576.

3. Curatolo P, Moavero R, de Vries PJ. Neurological and neuropsychiatric aspects of tuberous sclerosis complex. Lancet Neurol. 2015;14(7):733-745.

4. Krueger DA, Northrup H, International Tuberous Sclerosis Complex Consensus G. Tuberous sclerosis complex surveillance and management: recommendations of the 2012 International Tuberous Sclerosis Complex Consensus Conference. Pediatr Neurol. 2013;49(4):255-265.

5. Yates JR, Maclean C, Higgins JN, Humphrey A, le Marechal K, Clifford M, Carcani-Rathwell I, et al. The Tuberous Sclerosis 2000 Study: presentation, initial assessments and implications for diagnosis and management. Arch Dis Child. 2011;96(11):1020-1025.

6. Mizuguchi M, Takashima S. Neuropathology of tuberous sclerosis. Brain Dev. 2001;23(7):508-515.

7. von Ranke FM, Zanetti G, e Silva JL, Araujo Neto CA, Godoy MC, Souza CA, Mancano AD, et al. Tuberous sclerosis complex: state-of-the-art review with a focus on pulmonary involvement. Lung. 2015;193(5):619-627.

8. Hinton RB, Prakash A, Romp RL, Krueger DA, Knilans TK, International Tuberous Sclerosis Consensus G. Cardiovascular manifestations of tuberous sclerosis complex and summary of the revised diagnostic criteria and surveillance and management recommendations from the International Tuberous Sclerosis Consensus Group. J Am Heart Assoc. 2014;3(6):e001493.

9. Salerno AE, Marsenic O, Meyers KE, Kaplan BS, Hellinger JC. Vascular involvement in tuberous sclerosis. Pediatr Nephrol. 2010;25(8):1555-1561.

10. Jost CJ, Gloviczki P, Edwards WD, Stanson AW, Joyce JW, Pairolero PC. Aortic aneurysms in children and young adults with tuberous sclerosis: report of two cases and review of the literature. J Vasc Surg. 2001;33(3):639642.

11. Chaikof EL, Brewster DC, Dalman RL, Makaroun MS, Illig KA, Sicard GA, Timaran $\mathrm{CH}$, et al. The care of patients with an abdominal aortic aneurysm: the Society for Vascular Surgery practice guidelines. J Vasc Surg. 2009;50(4 Suppl):S2-49.

12. van Reedt Dortland RW, Bax NM, Huber J. Aortic aneurysm in a 5-year-old boy with tuberous sclerosis. J Pediatr Surg. 1991;26(12):1420-1422.

13. Rolfes DB, Towbin R, Bove KE. Vascular dysplasia in a child with tuberous sclerosis. Pediatr Pathol. 1985;3(24):359-373.

14. Jozwiak J, Jozwiak S, Oldak M. Molecular activity of sirolimus and its possible application in tuberous sclerosis treatment. Med Res Rev. 2006;26(2):160-180.

15. Franz DN, Leonard J, Tudor C, Chuck G, Care M, Sethuraman G, Dinopoulos A, et al. Rapamycin causes regression of astrocytomas in tuberous sclerosis complex. Ann Neurol. 2006;59(3):490-498.

16. Steagall WK, Taveira-DaSilva AM, Moss J. Clinical and molecular insights into lymphangioleiomyomatosis. Sarcoidosis Vasc Diffuse Lung Dis. 2005;22(Suppl 1):S4966.
17. Wienecke R, Fackler I, Linsenmaier U, Mayer K, Licht $\mathrm{T}$, Kretzler M. Antitumoral activity of rapamycin in renal angiomyolipoma associated with tuberous sclerosis complex. Am J Kidney Dis. 2006;48(3):e27-29.

18. Jones CT, Raman VT, DeVries S, Cole JW, Kelleher KJ, Tobias JD. Optimizing anticonvulsant administration for children before anesthesia: a quality improvement project. Pediatr Neurol. 2014;51(5):632-640.

19. Diaz JH. Perioperative management of children with congenital phakomatoses. Paediatr Anaesth. 2000;10(2):121128.

20. Cote CJ, Todres ID, Ryan JF, Goudsouzian NG. A practice of anesthesia for infants and children. 3rd ed. Philadelphia, Pa: WB Saunders; 2001:45, p. 184-185, 573.

21. Henretta JP, Hodgson KJ, Mattos MA, Karch LA, Hurlbert SN, Sternbach Y, Ramsey DE, et al. Feasibility of endovascular repair of abdominal aortic aneurysms with local anesthesia with intravenous sedation. J Vasc Surg. 1999;29(5):793-798.

22. Schwartz RA, Fernandez G, Kotulska K, Jozwiak S. Tuberous sclerosis complex: advances in diagnosis, genetics, and management. J Am Acad Dermatol. 2007;57(2):189202.

23. Rabito MJ, Kaye AD. Tuberous sclerosis complex: perioperative considerations. Ochsner J. 2014;14(2):229-239.

24. Lie JT. Cardiac, pulmonary, and vascular involvements in tuberous sclerosis. Ann N Y Acad Sci. 1991;615:58-70.

25. Matsumoto S, Nishioka T, Akiyama T. Renal angiomyolipoma associated with micronodular pneumocyte hyperplasia of the lung with tuberous sclerosis. Int J Urol. 2001;8(5):242-244.

26. Serpa Neto A, Schultz MJ, Gama de Abreu M. Intraoperative ventilation strategies to prevent postoperative pulmonary complications: Systematic review, meta-analysis, and trial sequential analysis. Best Pract Res Clin Anaesthesiol. 2015;29(3):331-340.

27. Hagood CO, Jr., Garvin DD, Lachina FM, Polsky WS, Ball TP, Bobroff LM. Abdominal aortic aneurysm and renal hamartoma in an infant with tuberous sclerosis. Surgery. 1976;79(6):713-715.

28. Wong H, Hadi M, Khoury T, Geary D, Rubin B, Filler G. Management of severe hypertension in a child with tuberous sclerosis-related major vascular abnormalities. J Hypertens. 2006;24(3):597-599.

29. Lavocat MP, Teyssier G, Allard D, Tronchet M, Freycon F. [Abdominal aortic aneurysm and Bourneville's tuberous sclerosis]. Pediatrie. 1992;47(7-8):517-519.

30. Al-Hashimi M, Thompson J. Anaesthesia for elective open abdominal aortic aneurysm repair. Cont Educ Anaesth Critical Care Pain. 2013;13:208-212.

31. Zammert M, Gelman S. The pathophysiology of aortic cross-clamping. Best Pract Res Clin Anaesthesiol. 2016;30(3):257-269.

32. Chaikof EL, Dalman RL, Eskandari MK, Jackson BM, Lee WA, Mansour MA, Mastracci TM, et al. The Society for Vascular Surgery practice guidelines on the care of patients with an abdominal aortic aneurysm. J Vasc Surg. 2018;67(1):2-77 e72. 